\title{
Effect of Garlic on Perfusion Scintigraphy of Rabbit's Lungs Following Experimentally-Induced Pulmonary Embolism
}

\author{
Gholamreza Assadnassab \\ Department of Clinical Science, Faculty of Veterinary Medicine, Tabriz Branch, Islamic Azad University, Tabriz, Iran
}

*For correspondence: Email: assadnassabgh@iaut.ac.ir; Tel: +98 9144121145

\begin{abstract}
Purpose: To study of the effect of garlic on rabbit's lungs, with the aid of perfusion scintigraphy, after experimentally-induced pulmonary embolism.

Methods: Twelve adult rabbits were anesthetized. Prepared macroaggregated albumin- technetium $99 \mathrm{~m}\left({ }^{99 m}\right.$ Tc-MAA) radiopharmaceutical was injected into the ear vein at a dose of $1.5 \mathrm{mCi}$. Perfusion scintigraphic images of the healthy rabbits' lungs were taken, and pulmonary embolism was experimentally induced by injection of autologous blood clots. Again, lung perfusion scintigraphic images of the rabbits' lungs were obtained. The animals were divided into two groups. The first group received normal saline and the other group received garlic tablet medication for seven days. Thereafter, perfusion scintigraphic images of the lungs were generated.

Results: The symmetric accumulation of radiotracer in the right and the left lobes of the lungs were observed in the scintgraphic images of the healthy animals. In embolic status scans, non-symmetric accumulation of the radiopharmaceutical was seen while in the scintigrams of garlic-treated group with pulmonary embolism, symmetric absorption of radioisotope was observed, similar to the perfusion lung scans of healthy animals.

Conclusion: The results obtained show that garlic may help to dissolve the blood clots that cause pulmonary embolism in rabbits.
\end{abstract}

Keywords: Garlic, Pulmonary embolism, Blood clots, Rabbit, Perfusion scintigraphy, Radioisotope

Tropical Journal of Pharmaceutical Research is indexed by Science Citation Index (SciSearch), Scopus, International Pharmaceutical Abstract, Chemical Abstracts, Embase, Index Copernicus, EBSCO, African Index Medicus, JournalSeek, Journal Citation Reports/Science Edition, Directory of Open Access Journals (DOAJ), African Journal Online, Bioline International, Open-J-Gate and Pharmacy Abstracts

\section{INTRODUCTION}

The diagnosis of respiratory system diseases, due to its vital role in the major functions, is highly important. A common and serious pulmonary disease is pulmonary emboli, which needs accurate and on-time diagnosis. Several techniques have been proposed for the diagnosis of pulmonary emboli one of which is the use of nuclear medicine imaging [1].

In this technique, after administration of special radioactive material into the body, the accumulation of the radiotracer in the target areas, in proportion to blood flow and its absorption, gets higher than the other areas. These regions are known as 'hot areas' [2].

In the areas with reduced intake or impaired absorption, there is less radiotracer uptake. These areas are called hypoactive or cold areas. By counting the radioactive material and defining the special pigments for the identified counts, images can be displayed on the monitor. In these scans, by recognizing and analyzing the hot and 
cold areas, a diagnostic image or a scintigram can be obtained [2].

In pulmonary embolism, because of narrow pulmonary arteries, the blood flow decreases, leading to a lower uptake of radiotracer in the affected area. In other words, embolism causes the creation of cold area in lung perfusion scan [2].

The obstruction of a large artery by a blood clot in the lung with a pulmonary embolism is a problem [1]. In nuclear medicine, for the diagnosis of pulmonary embolism, macroaggregated albumin- technetium 99m ${ }^{99 m}$ Tc-MAA) is injected into the patient's vein. After the injection, the large molecules of this material are first sent to the heart and then to the lungs. The large molecules are too large to pass through the pulmonary capillaries, so they are temporarily trapped at the entrance of some active veins. To differentiate between parenchymal lung disease (such as asthma or emphysema) and pulmonary embolism, lung ventilation scans can be performed using radioactive gases or radioaerosols [2]. Respiratory diseases also exist in animals, and the conflicts and side effects of the emboli are adverse pulmonary conditions affecting animals, too [3].

To treat emboli and reduce its side effects, several medications are used [1]. In this study, the medical effects of garlic during lung perfusion by nuclear medicine imaging has been studied.

Garlic has long had medical applications, the most prevalent of which, in recent years, has been used for the cardiovascular and antimicrobial effects [4]. Studies also show the significant effects of garlic on lowering the blood fat, its anti-platelet activity and anti-hypertensive feature $[4,5]$. Garlic has been used to reduce fat and atherosclerosis in animals, including rabbits [5].

In the present study, the effect of garlic administration was investigated in the scintigraphic perfusion of the rabbits' lungs following experimentally induced lung emboli. The scintigraphic perfusion images of the lungs before and after the garlic administration were compared, the infected parts were identified, and the healing process was examined.

\section{EXPERIMENTAL}

\section{Materials}

ADAC Gama camera (Vertex model, Philips Inc) and Capintec (model CRC-5 model, Capintec,
USA) dose calibrator machine were used. The macroaggregated albumin (MAA) kit (Kavoshyar, Iran), technetium-99m macroaggregated albumin ( ${ }^{99 m}$ Tc-MAA), and molybdenum-technetium generator used in the study were prepared and confirmed by the Atomic Energy Organization of Iran.

\section{Animals}

A total number of 12 mixed bred rabbits weighing $3.5 \pm 0.5 \mathrm{~kg}$ were selected. Then, the necessary blood and parasite analysis as well as clinical examinations were carried out. Once their health was confirmed, the needed antiparasitic drug (Ivermectin at a dose of $0.2 \mathrm{mg} / \mathrm{kg}$ ) was injected subcutaneously [6], and afterwards, the rabbits were provided for scintigraphic imagings.

This experimental study was approved by the Ethical Committee and Research Council of Tabriz Branch, Islamic Azad University, Tabriz, Iran (no. 13/11/5/11025). Examinations were carried out under the Ethical Committee guidelines as "Guide for the Care and Use of Laboratory Animals" [7] in the Faculty of Veterinary Medicine, Tabriz Branch, Islamic Azad University, Tabriz, Iran.

\section{Methods}

This study used an experimental treatment design. At the start of the experiment, the rabbits were anesthetized by acepromathine drug (1 $\mathrm{mg} / \mathrm{kg}$, Alfasan, Woerden, Holland), xylazine (Alfasan, Woerden, Holland) at a dose of 5 $\mathrm{mg} / \mathrm{kg}$ as well as pre-anesthetic medications, and ketamine anesthetic drug (35 mg/kg, Alfasan, Woerden, Holland) [8]. The anesthetized animals were laid spine recumbent on a scan table with stretched hands and feet. A detector of gamma camera was positioned under the table. A static scan had been examined for the existence of radioactive material.

The radioisotope, which was technetium, was milked from molybdenum- technetium generator and then added to macroaggregated albumin (MAA) kit. $1.5 \mathrm{mCi}$ of prepared ${ }^{99 \mathrm{~m}} \mathrm{TC}-\mathrm{MAA}$ was accumulated inside of an Insulin syringe by dose calibrator machine. Then it was quickly injected into the marginal ear vein. After injection, the syringe's secondary activity was measured by the same dose calibrator machine. A person must be careful to ensure that the animal did not move in order not to disrupt the scanning operation. For counting to be carried out efficiently, a lead shield was used around the injected ear. Such as another studied, this radiopharmaceutical passed from the healthy 
blood barrier and accumulated in proportion to the blood flow in the lungs $[2,3]$.

Five minutes after the injection of the radioactive material by regulating the scintillator of the apparatus, the scanning began. The scanning was carried out using the routine standard pulmonary scintigraphy imaging methods.

After the primary scanning operations, which are regarded as the necessary comparative images for comparing the subsequent scans, the animals were housed in standard polypropylene cages at a temperature of $24 \pm 3^{\circ} \mathrm{C}$ and with free access to food and water [7]. After this housing time (when the radioactive materials had completely been released from the animal's body), standard surgery operation was done and embolism in rabbits was established. The blood clots of the same animal were used to create the emboli which were then injected inside of the right jugular vein. Ji et al and Yang et al created lung emboli in all of the rabbits in their studies [9,10]. The blood from the animal was collected and maintained in an appropriate lab tube for one hour, and after this time, the clot was cut and prepared in small pieces (like Cui-Ping applied method with the dimensions of $2 \times 5 \mathrm{~mm}$ ) and was transferred to the vein [11].

In order to ensure the existence of emboli in the rabbits, the radioactive materials were injected via the ear after three days, and then the all animals were scanned by scintigraphy. After that, they were randomly divided into two groups with the same diet pattern. In each group, half of the animals were male and the other half were female. In the first group, six rabbits received orally garlic tablets $250 \mathrm{mg} / \mathrm{kg} /$ day (Daru Gol Herbal Drugs Incorporation, Iran) that dissolved in double-distilled water for one week [12]. A syringe and a gavage feeding tube (Aratebfan Co., Iran) were used in this purpose. In the second group, six rabbits served as the control group, which received normal saline by using a same syringe and gavage feeding tube. The volume of the drug and normal saline mixtures fed to the animals was $5 \mathrm{ml}$. Rabbits were allowed free access to similar nutritional diets. A week after the garlic administration, the animals were scanned using perfusion lung scintigraphy by injecting of ${ }^{99 m}$ Tc-MAA radiopharmaceutical as described above.

\section{Statistical analysis}

In all scans, accumulation of radiotracer in the right and the left lobes of lungs were compared using a standard visual qualitative gamma camera technique studies.

\section{RESULTS}

In this survey, the obtained images compared with them in the studies groups. The samples of scans in groups are shown in Figures 1-4.

The symmetric accumulation of radiotracer in the right and the left sides are observed in all healthy animals (Fig 1).

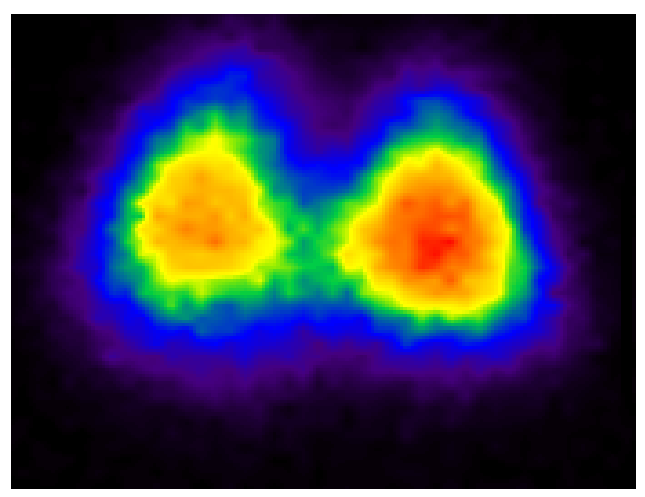

Figure 1: Scintigram of the lung in a healthy rabbit showing symmetric accumulation of radiotracer in the right and the left lobes of rabbit lung

The embolic status scans show non-symmetric accumulation of radiotracer in the right and the left lobes of lungs but the right side accumulation was lower than in the left side (Fig 2).

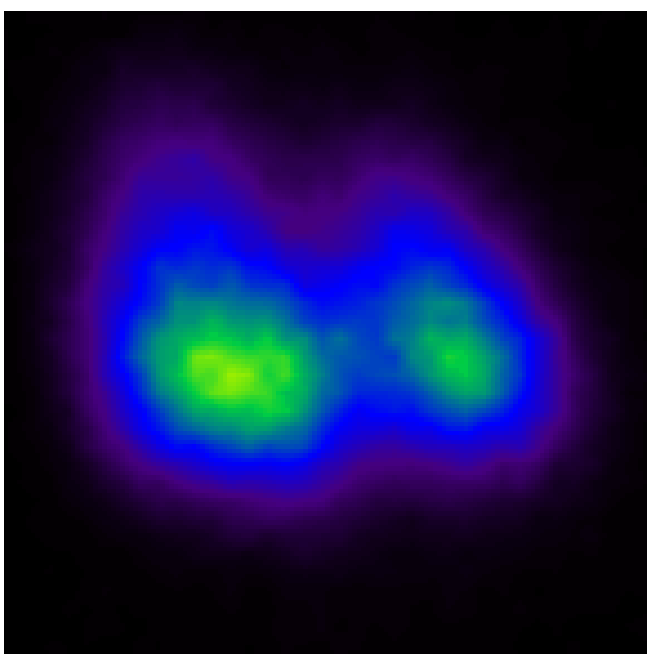

Figure 2: Perfusion lung scintigram in an embolic rabbit (injected with blood clots), in the right side accumulation of radiotracer is lower than the left side

In the pulmonary embolic scintigrams with feeding of normal saline have been observed again non-symmetric accumulation of radiotracer like as embolic perfusion lung scans (Fig 3). 


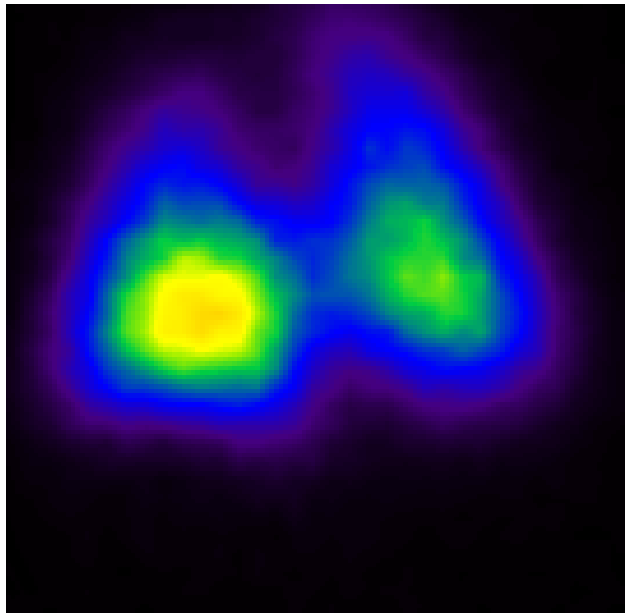

Figure 3: Scintigram of the lungs in an embolic rabbit (normal saline group), uptake of radiotracer is less in the right than the left side

In the perfusion lung scintigrams of embolic rabbits fed with garlic, symmetric accumulation of ${ }^{99 m}$ TC-MAA radiopharmaceutical in the right and the left sides was similar to healthy perfusion lung scans (Fig 4).

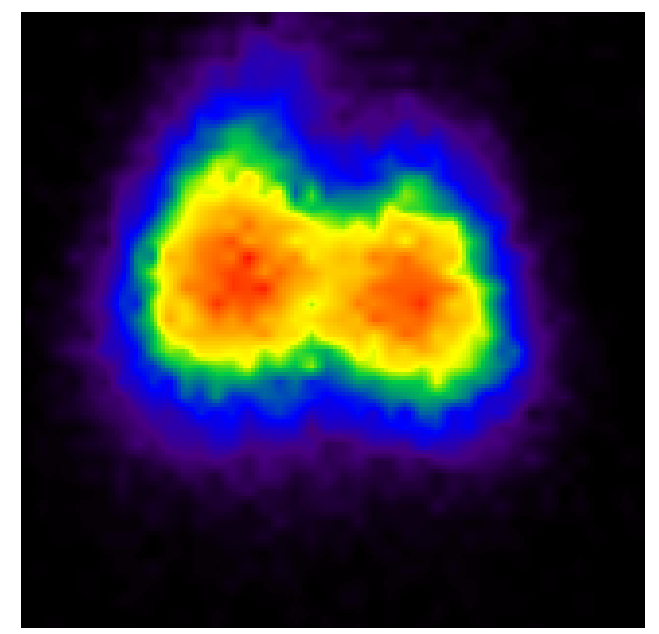

Figure 4: Perfusion lung scintigram of an embolic rabbit fed with garlic (garlic medication group); uptake of ${ }^{99 m} \mathrm{TC}-\mathrm{MAA}$ in the right side is similar to that for the left side

As the figures clearly show, the colored areas are red, yellow, and green. In scintigraphy, the greater the uptake of radiotracer by an organ is, the greater the radioactivity it will show, and the more colored it appears. In the images obtained, yellow shows greater uptake of the radiotracer in the lungs. However, the areas with less absorption of the radiopharmaceutical and lower radioactivity have lighter colors, represented as red and green, which are defined in advance in the test instrument.
Generally, the results show that absorption of radioisotope in healthy areas is higher than in embolic areas which is easily seen in the images.

\section{DISCUSSION}

Certain drugs are used for the treatment of pulmonary embolism. Nowadays, the effects of new drugs in this field are required and if approved will be added to the new treatment protocols. The current study is in line with this approach and shows the effect of garlic on induced low-level pulmonary embolism in rabbit. There are several ways to survey the condition of pulmonary embolism, one of which is the use of nuclear medicine imaging [1]. Perfusion pulmonary scintigraphy is considered an applied technique in nuclear medicine imaging. We can enable diagnosis of disease from normal condition by this technique [2,10]. Radioactive materials are used in diagnostic scanning, and one area in which they are most commonly used is the lung scan. The present study showed that radiopharmaceutical materials such as ${ }^{99 \mathrm{~m}} \mathrm{TC}$ MAA can be used for animals, especially rabbits, and this has been confirmed by other studies $[10,11]$.

The results are in agreement with the findings of Ji et al and Yang et al studies conducted on the inducing creation pulmonary emboli in rabbits $[9,10]$.

The results show that uptake of radioisotope in healthy areas is higher than in embolic areas which is easily seen in the images. Symmetric accumulation of radiotracer in the right and left sides were observed in healthy animals.

In the present study, embolic status scans show non-symmetric accumulation of radiotracer in the right and the left lobes of lungs and in the right side accumulation was lower than the left side of body, probably due to the anatomic structure and the point of injection. As for the other identified scans, the concentration of the radioactive materials was observed in active capillaries, and there was a lower level of radioactivity in the embolic areas of the lungs, caused by perfusion defect, in the diseased areas. This, too, is in agreement with the results of previous studies $[2,3,10]$.

Garlic (Allium sativum) has long been used for medical purposes, and it has recently been used for cardiovascular illnesses [12-14]. In animals, especially in rabbits and rats, garlic has been used for controlling and healing some diseases. For instance, it has been applied to rabbits for lowering fat and atherosclerosis [5,15]. 
In the perfusion lung scintigrams of embolic rabbits fed with garlic, symmetric accumulation of ${ }^{99 m}$ TC-MAA radiopharmaceutical in the right and left sides were observed to be similar to that of healthy perfusion lung scans but in other embolic rabbits fed normal saline, non-symmetric accumulation of radiotracer such as embolic perfusion lung scans. The findings of this study, indicate that garlic may serve as an effective anti-clotting medications that confirms the previous studies $[5,14]$.

Also, the scanned images indicate that garlic possesses anticoagulant effects, implying that garlic can be administered in hypertension cases. This provides further confirmation of previous studies in this regard $[4,12]$.

The results show that the garlic increased blood clot lysis in compared with control group which demonstrates fibrinolysis effect of garlic and this conforms to Ansari et al study [14].

The current study revealed further evidence for the effectiveness of garlic in atherosclerosis by dissolving of blood clots that formed of it $[14,15]$.

Due to the prevalence of pulmonary emboli and its side effects in humans, many studies can be carried out under different conditions (e.g., nutritional, environmental, temporal, etc) or other parameters such as new drugs administration [1]. The effects of other drugs and appropriate treatment methods can be explored by this technique, which is very valuable. These surveys can help find appropriate treatments for reducing the prevention of emboli in human societies. Clinical signs can be confirmed by identifying embolic areas.

\section{CONCLUSION}

The findings of this study indicate that administration of garlic as a medication may aid the dissolution of blood clots that cause pulmonary emboli. Further studies, including clinical tests, are required to confirm this effect in humans.

\section{ACKNOWLEDGEMENT}

The authors would like to thank Tabriz Branch, Islamic Azad University, Research Department, for financial support of this work, which is based on a research project contract, and Dr Dabiri Nuclear Medicine Center for their assistance.

\section{REFERENCES}

1. Acton $Q A$, Ed. Pulmonary Embolism: New Insights for the Healthcare Professional. Georgia: Scholarly Editions; 2013; pp 11-89, 118-122.

2. Mettler FA, Guiberteau MJ, Eds. Essentials of Nuclear Medicine Imaging. 6th edn. Philadelphia: Elsevier/Saunders; 2012; pp 195-250.

3. Zhang L, Chai X, Wu S, Zhao Y, Hu X, Hu Y, Xue, Y, Yang, G, Zhu, Hong Lu, G. Detection of pulmonary embolism by dual energy CT: correlation with perfusion scintigraphy and histopathological findings in rabbits. Eur Radiol 2009; 19(12): 2844-2854.

4. Grainger Bisset NG, Wichtl M, Eds. Herbal drugs and phytopharmaceuticals. 2nd edn. Boca Raton: CRC Press; 2001; pp 58, 293, 309, 312.

5. Durak I, Oztürk H, Olcay E, Can B, Kavutcu M. Effects of garlic extract on oxidant/antioxidant status and atherosclerotic plaque formation in rabbit aorta. Nutr Metab Cardiovasc Dis 2002; 12(3): 141-147.

6. Plumb DC, Ed. Plumb's Veterinary Drug Handbook. 4th edn. lowa: lowa State Press; 2005; pp 451-477.

7. Garber JC, Ed. Guide for the care and use of laboratory animals. Washington, D.C.: National Academies Press; 2010; pp 47-150, 193-217.

8. Flecknell PA, Ed. BSAVA manual of rabbit medicine and surgery. London: British Small Animal Veterinary Association; 2000; pp 30-31, 106.

9. Ji Y, Gao H, Zhang Z. Exploration of Rabbit Model of Pulmonary Embolism with Autologous Blood Clots. Lab Anim Sci Admin 2001; 4: 1-4.

10. Yang G, Yang X, Zhang L, Zhu H, Chai X, Hu X, Hu, Y, Lu, G. Pulmonary enhancement imaging with dual energy CT for the detection of pulmonary embolism in a rabbit model: comparison to perfusion planar scintigraphy, SPECT and SPECT-CT modalities. Acad Radiol 2011; 18(5): 605-614.

11. Cui-ping LV, Yang $H$, Dong $X$. Establishment of rabbit's acute pulmonary thromboembolism model and measurement of right ventricle tension and electrocardiogram. Chin J Comp Med 2009; 11(83): 27-30.

12. Banerjee SK, Maulik SK, Eds. Effect of garlic on cardiovascular disorders: A review. Nutrit J 2002; 1(1): 4.

13. Gebreyohannes G, Gebreyohannes M. Medicinal values of garlic: A review. Int J Med Med Sci 2013; 5(9): 401-408.

14. Ansari F, Soltan Mohammadi N, Naderi G, Sadegh Sabet $M$, Karimi A. A Study of garlic effect on fibrinolytic activity of the blood clot in vitro. Iran J Ped Hematol Oncol 2011; 1(2): 48-55.

15. Durak Ä, Öztürk HS, Olcay E, Guven C. Effects of garlic extract supplementation on blood lipid and antioxidant parameters and atherosclerotic plaque formation process in cholesterol-fed rabbits. J Herb Pharmacother 2002; 2(2): 19-32. 